\title{
Wahrgenommene Zielkonflikte zwischen Gesundheitszielen: Ergebnisse einer Intervention zur Förderung von körperlicher Aktivität und Ernährung
}

\author{
Perceived intergoal conflicts between two health goals: Results of a health prevention to enhance physical \\ activity and a healthy diet
}

Axinja Hachfeld ${ }^{1 *}$, Sonia Lippke ${ }^{2,3}$, Jochen P. Ziegelmann ${ }^{3} \&$ Alexandra M. Freund ${ }^{4}$

\begin{abstract}
Zusammenfassung
Fragestellung: Ungesunde Ernährung und körperliche Inaktivität gehören zu den Hauptursachen vieler Krankheiten. Aus diesem Grund ist die Entwicklung von Präventionsmaßnahmen und Gesundheitsförderung wichtig, die sowohl gesunde Ernährung als auch körperliche Aktivität fördern. Gesundheitsförderung, die an mehreren Gesundheitszielen ansetzt, kann Teilnehmende jedoch überfordern. Ziel der Studie war es deshalb, (a) den $\mathrm{Zu}$ sammenhang dieser beiden Gesundheitsverhaltensweisen zu untersuchen, (b) die wahrgenommenen Zielkonflikte und Zielkohärenzen zu erheben, und (c) die Auswirkung von stadienpassender und stadienunpassender Gesundheitsförderung auf Zielkonflikt und Zielkohärenz zu testen.

Methode: Auf Grundlage des Sozial-kognitiven Prozessmodells des Gesundheitsverhaltens (Health Action Process Approach: HAPA, Schwarzer, 2008) wurde in der vorliegenden Studie eine internetbasierte Intervention zur Förderung von körperlicher Aktivität und gesunder Ernährung entwickelt. Das HAPA unterscheidet verschiedene Stadien des Gesundheitsverhaltens, die bei der Evaluation der verschiedenen Interventionen berücksichtigt wurden. Die quasi-experimentelle Studie wurde an $N=1260$ und einer längsschnittlichen Substichprobe von $n=300$ durchgeführt.

Ergebnisse: Die Ergebnisse zeigten, dass die ernährungs- und bewegungsbezogenen Gesundheitsziele positiv korreliert sind, und die Teilnehmenden mehr Kohärenz als Konflikte berichteten. Die wahrgenommenen Konflikte zwischen den Gesundheitszielen unterscheiden sich in den verschiedenen HAPA-Stadien. Stadienpassende Interventionen reduzierten Zielkonflikte signifikant.

Schlussfolgerung: Gesundheitsförderung, die gleichzeitig bei Bewegung und Ernährung ansetzt, scheint die Teilnehmenden nicht zu überfordern. Im Gegenteil, Teilnehmende berichten eine hohe Kohärenz zwischen beiden Gesundheitszielen. Allerdings spielen Konflikte besonders zu Beginn der Verhaltensänderung und bei der Intentionsbildung eine zentrale Rolle. Gesundheitsförderungsprogramme sollten dies berücksichtigen. Interventionen können solche Konflikte reduzieren.
\end{abstract}

\section{Schlagworte}

Körperliche Aktivität, Ernährung, Zielkonflikte, Gesundheitsförderung

\begin{abstract}
Objectives: Unhealthy diet and physical inactivity are main causes of many diseases and hence, the development of effective interventions is warranted. However, targeting both health behaviors simultaneously might overburden participants. Thus, the aim of the study was to (a) investigate the relationship between these two health behaviors, (b) assess the perceived intergoal conflict and coherence, and (c) test the effects of stage-matched and mismatched interventions on perceived intergoal conflict and coherence.

Methods: Based on the Health Action Process Approach (HAPA; Schwarzer, 2008), which describes behavior change as a process with qualitatively different stages, an internet-based health prevention program was tested. The quasi-experimental study with two different interventions included $N=$ 1260 and a longitudinal subsample with $n=300$ participants.

Results: Results showed that both health behaviors are correlated, and that participants perceived more intergoal facilitation than conflicts. Perceived conflict varied between participants in the different HAPA stages. Stagematched interventions successfully reduced intergoal conflict.

Conclusion: Health promotion programs that simultaneously target physical activity and dietary behavior seem not to overburden participants. On the contrary, participants report high levels of intergoal coherence between the two goals. Nonetheless, intergoal conflicts do play a role in the early stages of behavior change and intention formation. Health promotion programs should take these results into consideration when more than one health behavior is to be targeted.
\end{abstract}

Key-Words

Physical activity, dietary behavior, intergoal conflicts, health promotion
Korrespondenzadresse: Axinja Hachfeld, Max Planck Institut für Bildungsforschung, Lentzeallee 94, 14195 Berlin. Tel. +49 (30) 82406671; E-mail: hachfeld@mpib-berlin.mpg.de.

1 Max Planck Institut für Bildungsforschung.

2 Universiteit Maastricht, The Netherlands.

3 Freie Universität Berlin.

4 Psychologisches Institut, Universität Zürich.

* Die Autorin hat bereits unter dem Namen Axinja Kalusche zu dem Thema publiziert.

Anmerkung der Autoren:

Wir möchten Lena Fleig für die hilfreichen Rückmeldungen zu einer früheren Version des Manuskriptes danken. Jana Richert danken wir für die Unterstützung bei der Datenerhebung.

\section{Einleitung}

Ungesunde Ernährung und körperliche Inaktivität gehören zu den Hauptursachen vieler Krankheiten. So zählt beispielsweise Übergewicht zu einem der größten Gesundheitsprobleme in Deutschland, auf das ein Großteil der Krankheitsbelastung zurückzuführen ist (Robert Koch-Institut, 2006) und das sich durch gesündere Ernährung und Bewegung stark reduzieren ließe. Doch auch Normalgewichtige profitieren gesundheitlich durch ein verbessertes Ernährungs- und Bewegungsverhalten. Aus diesem Grund setzen verschiedene Ge- 
sundheitsförderungsmaßnahmen ${ }^{1}$ an diesen beiden Punkten an (Kniesel et al., 2007).

Gesundheitsförderung ist kostspielig und es stellt eine Herausforderung dar, einen möglichst großen Anteil aller Betroffenen erreichen zu können (Stetina \& Kryspin-Exner, 2009). Um dies kostengünstig zu realisieren, müssen neue Wege für solche Maßnahmen gefunden werden. Solch einen neuen Weg bietet das Internet (Stetina \& Kryspin-Exner, 2009). Umfragen zufolge verfügen bereits über $60 \%$ der deutschen Haushalte über einen Internetzugang (vergleiche Schaubild 2, Ottens, 2005). Zunächst ist es jedoch notwendig, evidenz- und theoriebasierte Maßnahmen zur Gesundheitsförderung für dieses Medium zu entwickeln. Die vorliegende Studie untersucht einen theoriebasierten Ansatz, auf dessen Grundlage internetbasierte Interventionen in Zukunft aufgebaut werden könnten.

\subsection{Das Sozial-kognitive Prozessmodell des Gesundheitsverhaltens}

Theoretische Grundlage der vorliegenden Interventionsstudie war das Sozial-kognitive Prozessmodell des Gesundheitsverhaltens (Health Action Process Approach; HAPA; Schwarzer, 2008). Das HAPA beschreibt die Änderung eines Gesundheitsverhaltens als einen dynamischen Prozess: Menschen, die ihr Gesundheitsverhalten ändern möchten, gehen durch qualitativ verschiedene, aufeinander aufbauende Stadien. In jedem Stadium sind unterschiedliche sozial-kognitive Faktoren wie beispielsweise Risikowahrnehmung oder Planung bedeutsam und entscheidend für den Wechsel in das nächste Stadium. Am Ende dieses Prozesses steht die eigentliche Verhaltensänderung. Das HAPA unterscheidet dabei drei Stadien. Im Rahmen von Stadienmodellen sind Interventionsmaßnahmen dann erfolgreich oder effektiv, wenn diese zu einem Stadienaufstieg und letztendlich hin zur Ausübung des gewünschten Gesundheitsverhaltens führen (vergleiche Wiedemann et al., 2009). Zahlreiche Befunde haben gezeigt, dass Interventionen dann effektiver sind, wenn sie auf das jeweilige Stadium des Betroffenen zugeschnitten werden (Noar et al., 2007; Weinstein et al., 1998; Weinstein, Rothman \& Sutton, 1998). Dieses „Tailoring“ (maßschneidern) auf die Bedürfnisse der Teilnehmenden bringt zudem Kosten- und Zeitersparnis mit sich, da Interventionen sich auf die für das Stadium bedeutsamen Faktoren konzentrieren können. Zwar muss zu Beginn der Intervention eine Zuordnung zu einem Stadium erfolgen, allerdings geht diese Zuordnung mithilfe bereits vorhandener und validierter Algorithmen sehr schnell. Welche Faktoren für welche Stadi-

\footnotetext{
1 Als Gesundheitsförderung oder Präventionen werden in diesem Artikel all diejenigen (meist large-scale) Maßnahmen verstanden, die durchgeführt werden, um Gesundheit aufrechtzuerhalten. Der Begriff Intervention wird nur im engen, experimentellen beziehungsweise wissenschaftlichen Sinne verwendet. Die vorliegende Studie beinhaltet eine Intervention, die gegebenenfalls in Gesundheitsförderung oder Präventionsmaßnahmen überführt werden kann.
}

en besonders bedeutsam sind, wurde im Rahmen der verschiedenen Modelle bereits empirisch getestet. Verschiedene Studien haben die Effekte von stadienpassenden Intervention mit den Effekten einer Kontrollgruppe (Napolitano et al., 2003), einer Placebo- Intervention (Patrick et al., 2006) oder einer stadienunpassenden Intervention (Bolognesi et al., 2006; Fleig et al., 2010) verglichen. Zieht man als Kriterium Stadienwechsel heran, so konnte die Überlegenheit von stadienpassenden Interventionen in Übersichtsarbeiten gezeigt werden (vergleiche Bridle et al., 2005; Noar et al., 2007; Noar et al., 2009).

Das HAPA bestimmt das Stadium der Teilnehmenden aufgrund ihres derzeitigen Verhaltens und ihrer Änderungsabsicht (Zielintention, zum Beispiel ist der Teilnehmende schon körperlich aktiv? hat er die Absicht, aktiv zu werden beziehungsweise zu bleiben? vergleiche Lippke et al., 2009). $\mathrm{Zu}$ Beginn, im non-intentionalem Stadium, üben die Personen das Verhalten noch nicht aus und wollen dies auch nicht tun (Personen in diesem Stadium werden als Non-Intender kategorisiert, da es um den Aufbau einer Zielintention geht). Hier sind besonders Aspekte der Risikowahrnehmung bedeutsam, die auf die Intentionsbildung wirken. Eine Risikointervention wäre stadienpassend und demnach besonders effektiv für Personen in diesem Stadium. Das nächste Stadium (intentionales Stadium) ist erreicht, wenn eine Zielintention zur Verhaltensänderung gebildet wurde. Personen in diesem Stadium haben die Intention, ihr Verhalten zu ändern, sind aber noch nicht aktiv und werden als Intender bezeichnet. Hier ist es wichtig, mithilfe von konkreten Planungen die Intentionen in Verhalten umzusetzen. Lippke, Ziegelmann \& Schwarzer (2004) konnten zeigen, dass eine Planungsintervention in diesem Stadium erfolgreich körperliche Aktivität erhöhen kann. Erst im letzten Stadium (aktives Stadium) wird das Verhalten ausgeübt. Hier ist es wichtig, Personen (sogenannte Actor) dabei zu unterstützen, ihr Verhalten aufrechtzuerhalten.

Ein Aspekt, der beeinflussen kann, ob eine Person eine Zielintention aufbaut, umsetzt oder aufrechterhält, ist der des Zielkonfliktes (vergleiche Riediger \& Freund, 2004). Dies ist insbesondere bei mehreren (multiplen) Gesundheitszielen von Relevanz. Bisher werden Zielkonflikte aber noch nicht explizit in Stadienmodellen berücksichtigt und untersucht. Ein Ziel der vorliegenden Studie ist es, Erkenntnisse aus beiden Forschungsrichtungen zu verbinden. Im Folgenden soll zunächst auf Forschung zu Zielkonflikten eingegangen und diese dann mit den Annahmen des HAPA-Modell verknüpft werden.

\subsection{Multiple Gesundheitsziele}

Menschen verfolgen in ihrem täglichen Leben meist (mehrere) verschiedene Ziele. Diese Ziele können sich gegenseitig fördern (,Zielkohärenz“) oder behindern (,Zielkon$\left.f l i k t^{*}\right)$. Beispielsweise fördern sich die Ziele ,mehr Zeit in 
der Natur verbringen“ und „mit meinen Kindern Fahrradfahren üben“ gegenseitig. Anders die Ziele ,viel Sport treiben“ und „mehr Präsenz auf der Arbeit zeigen“, die miteinander in Konflikt stehen. Riediger \& Freund (2004; siehe auch Riediger et al., 2005) untersuchten in einer Studie Personen, die sich zum Ziel gesetzt hatten, mit regelmäßigem Sporttreiben anzufangen. Dabei zeigte sich, dass sich Konflikte zwischen dem Ziel regelmäßig Sport zu treiben und einem weiteren nicht-gesundheitsbezogenem Ziel vor allem negativ auf die emotionale Befindlichkeit auswirken, während Zielkohärenz die Zielerreichung im Alltag (hier: regelmäßiges Sporttreiben über einen Zeitraum von drei Monaten) fördert. Dieser Befund hat auch Implikationen für Gesundheitsförderung, die mehrere Gesundheitsziele anspricht.

Schafft es jemand nicht, seine verschiedenen Gesundheitsziele umzusetzen, könnte dies also auch daran liegen, dass diese Ziele eine geringe Kohärenz aufweisen oder miteinander konfligieren. Gesundheitsförderung, die mehr als ein Gesundheitsverhalten anspricht, wäre dann effektiver, wenn mögliche Konflikte und Kohärenzen zwischen den verschiedenen Gesundheitszielen berücksichtigt und reduziert würden. Wie eingangs geschildert, setzt Gesundheitsförderung meist sowohl bei Ernährung als auch bei körperlicher Aktivität an. Kritiker dieses Ansatzes weisen jedoch darauf hin, dass Gesundheitsmaßnahmen, die mehrere Verhaltensweisen gleichzeitig einbeziehen, die Teilnehmenden überfordern könnten (Prochaska \& Sallis, 2004). Andere Forscher sprechen demgegenüber von „synergistischen Effekten“ eines solchen Vorgehens (Vandelanotte et al., 2005). Von synergistischen Effekten wird zum Einen ausgegangen, wenn die positive Wirkung einer Intervention sich auch in anderen Verhaltensweisen, die nicht von der Intervention betroffen waren, niederschlägt. Zum anderen handelt es sich um synergistische Effekte, wenn die Wirkung einer Intervention dann stärker ist, wenn sie an zwei oder mehreren Verhaltensweisen ansetzt. Bisher ist ungeklärt, wie solche synergistischen Effekte entstehen und unterstützt werden können. Die oben beschriebene Forschung zu persönlichen Zielen und insbesondere zu Zielkonflikten bietet einen möglichen Erklärungsansatz. Die vorliegende Studie erfasst Zielkonflikte und Zielkohärenz zwischen den beiden Zielverhalten explizit und untersucht im Rahmen eines stadienpassenden Interventionsansatzes, ob die beiden Gesundheitsverhaltensweisen (körperliche Aktivität und gesunde Ernährung) sich eher gegenseitig fördern (Kohärenz) oder behindern (Konflikt).

Zielkonflikte stehen der Umsetzung von Zielen im Wege. Eine Reduzierung von Konflikten ist daher erstrebenswert, um die Wahrscheinlichkeit einer Umsetzung des gewünschten Verhaltens beziehungsweise eines Stadiumwechsels zu erhöhen. In diesem Sinne wären anfängliche Zielkonflikte ähnlich wie der Rubikon, den es zu überwinden gilt, bevor der nächste Schritt gegangen wird (vergleiche das
Rubikonmodell von Heckhausen \& Kuhl, 1984). Sind Zielkonflikte nicht in allen Stadien gleichermaßen stark ausgeprägt, könnten stadienpassende Interventionen effizient auf mögliche Konflikte eingehen. Im Rahmen von Interventionen können verschiedene Wege eingeschlagen werden, um Zielkonflikte zu reduzieren. So können mögliche Konflikte beispielsweise thematisiert und aktiv an der Lösung gearbeitet werden. Gleichzeitig haben Studien die Wirksamkeit von stadienspezifischen Interventionen gezeigt.

Demnach sind stadienpassende Interventionen besser als unpassende in der Lage, die für eine Verhaltensänderung in dem jeweiligen Stadium relevanten Variablen zu beeinflussen. In diesem Sinne wäre es auch denkbar, dass stadienpassende Interventionen Zielkonflikte auch dann effektiv reduzieren, wenn sie Konflikte nicht explizit thematisieren. Der Zusammenhang von stadienspezifischen Interventionen und Zielkonflikten wurde bisher jedoch noch nicht untersucht, daher ist das Vorgehen unserer Studie explorativ.

Wirken stadienspezifische Interventionen nur in Abwesenheit von Zielkonflikten oder sind stadienspezifische Interventionen mehr als stadienunpassende Interventionen in der Lage, Zielkonflikte sogar dann zu reduzieren, wenn diese nicht explizit im Rahmen der Intervention thematisiert werden? Wenn dies der Fall ist, wären stadienspezifische Interventionen auch oder sogar gerade dort einsetzbar, wo an mehreren Verhaltensweisen angesetzt werden muss. Vor allem, wenn davon auszugehen ist, dass die meisten Menschen sich hinsichtlich verschiedener Gesundheitsverhaltensweisen nicht in demselben Stadium befinden, sprich stadieninkongruent sind (vergleiche Boudreaux et al., 2003). Dies kann zum Beispiel bei der Diabetes-Prävention der Fall sein, für die körperliche Aktivität ebenso wichtig ist wie eine gesunde Ernährung.

Insgesamt wird erwartet, dass die meisten Personen die beiden Ziele als förderlich ansehen, da beide demselben übergeordneten Ziel einer gesunden Lebensweise dienen und daher nicht in einem Ressourcenkonflikt stehen oder inkompatible Zielverfolgungsstrategien implizieren (zu Quellen für Zielkonflikte siehe auch Riediger et al., 2005). Es ist jedoch auch möglich, dass sich die beiden Verhaltensbereiche behindern: Personen könnten beispielsweise durch eine Steigerung der körperlichen Aktivität mehr Hunger oder Appetit auf kohlenhydratreiche Nahrungsmittel entwickeln und daher befürchten, dass eine Steigerung ihrer körperlichen Aktivität ihre gesunde Ernährung behindern könnte. Umgekehrt könnten Personen befürchten, dass sie weniger Energie haben, wenn sie ihre Ernährung zum Beispiel auf mehr Verzehr von Obst und Gemüse umstellen und sich dann schwerer mit körperlichen Aktivitäten tun. Falls solche Zielkonflikte auftreten, würde das Gesundheitsförderung infrage stellen, die mehrere Gesundheitsverhalten gleichzeitig (simultan) anspricht. In diesem Fall wäre ein sequentielles Vorgehen vorzuziehen. 
Ein weiteres Ziel dieser Studie war es zu untersuchen, in welchen HAPA-Stadien Zielkonflikte besonders häufig sind, damit in diesen Stadien Zielkonflikte bei zukünftigen Gesundheitsförderungsmaßnahmen explizit berücksichtigt werden.

\subsection{Hypothesen}

Im Einzelnen wurden in dieser Studie folgende Hypothesen untersucht:

1. Die Intentionen, körperliche Aktivität auszuüben und Obst- und Gemüse zu essen, sind positiv miteinander korreliert. Die Ausübung körperlicher Aktivität und der regelmäßige Verzehr von Obst- und Gemüse sollten ebenfalls miteinander korreliert sein.

2. Die beiden Gesundheitsziele fördern sich gegenseitig (Kohärenz), das heißt Teilnehmende berichten häufiger, dass ihre körperliche Aktivität ihre gesunde Ernährung fördert als dass sie sie hemmt und, anders herum, dass ihre gesunde Ernährung ihre körperliche Aktivität mehr fördert als dass sie sie hemmt.

3. Personen im non-intentionalen Stadium nehmen mehr Konflikte und weniger Kohärenz zwischen den Gesundheitszielen wahr als Personen im intentionalen Stadium oder Personen im aktivem Stadium.

4. Teilnehmende in einer stadienpassenden Intervention erleben vom ersten zum zweiten Messzeitpunkt eine stärkere Abnahme ihres wahrgenommenen Zielkonflikts als Teilnehmende in stadienunpassenden Interventionen.

\section{Methode}

\subsection{Rekrutierung und Datenerfassung}

Zur Rekrutierung der Teilnehmenden gaben die Autoren Interviews in verschiedenen Zeitungen und Radiosendern. Die Rekrutierung erfolgte hauptsächlich durch nationale Presse und durch Verlinkung der Studie auf deutschsprachigen Internetseiten. Durch dieses Vorgehen wurde eine Stichprobe rekrutiert, die aus dem gesamten Bundesdeutschen Raum stammt, mit Schwerpunkt jedoch in der Region BerlinBrandenburg. Interessierte konnten auf die Internetseite gehen und dort den Fragebogen (inklusive der Intervention) ausfüllen. Der Fragebogen war auf der Homepage einer gesundheitspsychologischen Forschungsabteilung geschaltet und frei zugänglich. Die Internetseite klärte auch über das Forschungsvorhaben und die Rechte der Teilnehmenden auf. Am Ende des Fragebogens wurden die Teilnehmenden nach ihrer E-Mail-Adresse gefragt, um vier Wochen später zur zweiten Befragung eingeladen werden zu können. Die Angabe der E-Mail-Adresse war freiwillig, alle Angaben der Teilnehmenden wurden vertraulich behandelt. Die Teilnehmenden konnten ihre Teilnahme jederzeit widerrufen. Es wurde allen Teilnehmenden zur ersten Befragung mitgeteilt, dass diejenigen, die an beiden Messzeitpunkten teilnehmen, automatisch an einer Verlosung von Einkaufsgutscheinen in Höhe von 15 bis 30 Euro teilnehmen. Die Einladungsemail für den zweiten Teil enthielt den Link zur Internetseite mit der Befragung. Mit Hilfe des Programms dynQuest (Rademacher \& Lippke, 2007) wurden die Seiten gesteuert und die Eingaben gespeichert. Die Daten wurden anschließend in SPSS eingelesen und die E-Mail-Adresse separat von den Daten gespeichert. Unabhängig von der Interventionsgruppe hatten alle Teilnehmenden nach der Befragung die Möglichkeit, sich auf der Internetseite über den Vorteil von regelmäBiger körperlicher Bewegung und gesunder Ernährung zu informieren.

\subsection{Stichprobe}

Insgesamt nahmen $N=1260$ Personen an der ersten OnlineBefragung teil. Alle Teilnehmenden erhielten vier Wochen nachdem sie den ersten Fragebogen ausgefüllt hatten eine elektronische Einladung zur Teilnahme am zweiten Fragebogen. Die Einladungen wurden individuell für jeden Teilnehmenden verschickt. Die Teilnehmenden der ersten Befragung wurden für die Analysen zu den Hypothesen 1 - 3 herangezogen. Für die Überprüfung der längsschnittlichen Hypothese 4 wurden die Teilnehmenden herangezogen, die in der ersten Woche nach Freischaltung den ersten Fragebogen und vier Wochen später den zweiten Fragebogen beantwortet hatten $(n=300)$.

Zum ersten Messzeitpunkt waren die Teilnehmenden im Durchschnitt 40 Jahre alt $(S D=12.39$, Alter zwischen 16 und 80 Jahren) und $67 \%(n=789)$ waren weiblich. 59\% $(n=696)$ der Teilnehmenden waren verheiratet oder in einer festen Beziehung und $41 \%(n=513)$ hatten Kinder. Drei Viertel der Teilnehmenden hatten Abitur, über die Hälfte einen Hochschulabschluss (57\%). Zum Zeitpunkt der Erhebung waren $64 \%$ berufstätig. Die Teilnehmenden kamen aus dem gesamten Bundesgebiet, allerdings mit einem Schwerpunkt in Berlin und Umgebung (54\%). Der durchschnittliche Body Mass Index (BMI) lag bei $24.92(S D=5.20)$ für die weiblichen Teilnehmerinnen und bei $26.51(S D=5.02)$ für die männlichen und war damit sowohl für die Frauen als auch für die Männer im oberen Bereich des Normalgewichts (WHO, 2000). Allerdings hatten knapp die Hälfte aller Teilnehmenden einen BMI über 25 (43\% der Gesamtstichprobe, 38\% der Frauen und $55 \%$ der Männer) und gelten somit als übergewichtig (davon hatten $17 \%$ einen BMI von über 30, was sehr starkem Übergewicht entspricht). Die Teilnehmenden beim zweiten Messzeitpunkt unterschieden sich hinsichtlich der erneut erfassten sozio-demographischen Variablen nicht vom ersten Messzeitpunkt (68\% $(n=186)$ Frauen, durchschnittlich 40 Jahre, $S D=12.58$ und durchschnittlicher BMI 25.10, $S D=5.41)$. 


\subsection{Messinstrumente}

$\mathrm{Zu}$ beiden Messzeitpunkten wurden sozial-kognitive Variablen wie Intention und Planung, sowie das Verhalten der Teilnehmenden in den Bereichen körperliche Aktivität und Ernährung erfasst. Der Bereich Ernährung beschränkte sich auf den Obst- und Gemüseverzehr, da in letzter Zeit Gesundheitsorganisationen und Regierungen zu so genannten ,5am-Tag“-Kampagnen aufgerufen haben, das heißt fünf Portionen Obst und Gemüse (oder zirka $650 \mathrm{~g}$ ) sollen pro Tag konsumiert werden (vergleiche Deutsche Gesellschaft für Ernährung, 2004 und www.fuenfamtag.de). Des Weiteren wurden das Stadium (für körperliche Aktivität und Obst- und Gemüseverzehr: Non-intender, Intender, Actor), Zielkonflikt und -kohärenz sowie sozio-demografische Angaben erhoben. Alle Items wurden in vorherigen Studien validiert (Lippke et al., 2009; Riediger \& Freund, 2004; Schwarzer, 2008), wobei jedoch die Items zum Bereich Zielkonflikt und -kohärenz an die aktuelle Studie angepasst wurden.

\subsection{Intentionen}

Körperliche Aktivität. Die Intention, körperlich aktiv zu sein, wurde mit drei Items in Anlehnung an die Godin-Skala (Godin Leisure-Time Exercise Questionnaire; Godin \& Shepard, 1985) erfasst. Die Items lauteten: Ich habe mir vorgenommen, mindestens an fünf Tagen pro Woche 30 Minuten (a) anstrengende körperliche Aktivität auszuüben (Herz schlägt schneller, Schwitzen), (b) mittelmäßig anstrengend körperlich aktiv zu sein (nicht ermüdend, leichtes Schwitzen), (c) leicht körperlich aktiv zu sein (kaum Anstrengung, kein Schwitzen). Die Items wurden getrennt analysiert (vergleiche Lippke et al., 2009). Antworten konnten auf einer vier-stufigen Likert-Skala (stimmt nicht - stimmt genau) gegeben werden.

Ernährung. Die Intention, fünf Portionen Obst und Gemüse täglich zu essen, wurde mit zwei Items erhoben. Der Itemstamm war jeweils „Ich habe mir konkret vorgenommen ...". Die Items lauteten (a) ... mindestens fünf Portionen Obst und Gemüse täglich zu essen, und (b) ... zu jeder Mahlzeit Obst und Gemüse zu essen (vergleiche Schwarzer, 2008). Antworten konnten auf einer vier-stufigen LikertSkala (stimmt nicht - stimmt genau) gegeben werden. Für die Analysen wurden die Antworten zu beiden Fragen zu Obstund Gemüseverzehr zusammengefasst.

\subsection{Verhalten}

Körperliche Aktivität. Parallel zu den Intentionen wurde körperliche Aktivität in Anlehnung an die Godin-Skala erhoben. Die Teilnehmenden gaben an, wie häufig (in Minuten pro Woche) sie im letzten Monat neben ihrer körperlichen Aktivität im Alltag noch zusätzlich in ihrer Freizeit körperlich aktiv waren. Dabei sollten sie zwischen leich- ter, mittelmäßiger und anstrengender Aktivität unterscheiden. Leichte körperliche Aktivität war definiert durch keine erhöhte Anstrengung und kein Schwitzen (zum Beispiel leichtes Gehen), mittelmäßige körperliche Aktivität durch keine erhöhte Anstrengung und leichtes Schwitzen (zum Beispiel langsames Radfahren) und anstrengende körperliche Aktivität durch erhöhte Anstrengung und Schwitzen (zum Beispiel Fußballspielen). Um ein Maß für das Verhalten zu bilden, wurden die Antworten zu mittelmäßig anstrengender und anstrengender körperlicher Aktivität zu einem WochenSummenscore zusammengefasst (vergleiche Lippke et al., 2009).

Ernährung. Obst- und Gemüseverzehr wurde mit einem Item in Anlehnung an Rafferty und Kollegen (2002) erhoben. Die Teilnehmenden gaben auf einer vier-stufigen Likert-Skala an, ob sie bereits fünf Portionen Obst und Gemüse täglich essen (stimmt nicht - stimmt genau). Die Antwort auf dieses Item wurde für die Analysen verwendet. Für deskriptive Zwecke wurden die Teilnehmenden zusätzlich gebeten anzugeben, wie viele Portionen Obst und Gemüse sie in der letzten Woche täglich gegessen haben.

\subsection{Stadien}

Zur Erhebung der Stadien wurde ein Algorithmus verwendet, der die Personen in drei Stadien einteilt (Lippke et al., 2009). Der Algorithmus bezog dabei sowohl das Verhalten einer Person, als auch ihre Intention das Verhalten auszuüben ein. Zuerst wurden all diejenigen Personen, die noch keine Intention haben, das Zielverhalten auszuüben (entweder körperliche Aktivität oder regelmäßiger Obst- und Gemüseverzehr) als Non-Intender klassifiziert. Der zweite Schritt bezieht all diejenigen Personen ein, die bereits die Intention haben, das Zielverhalten auszuüben und unterscheidet diese hinsichtlich ihres tatsächlichen Verhaltens. Menschen, die bereits die Entscheidung getroffen haben, ihr Verhalten zu ändern, dies aber noch nicht in die Tat umgesetzt haben, werden dabei als Intender diagnostiziert. Personen, die die Handlung bereits ausüben, werden als Actors bezeichnet. Das Stadium wurde für jeden Teilnehmenden sowohl für körperliche Aktivität als auch für Obst- und Gemüseverzehr erhoben.

\subsection{Zielkonflikt und Zielkohärenz}

Zielkonflikt und Zielkohärenz zwischen den Zielen körperliche Aktivität und gesunde Ernährung wurden in Anlehnung an Riediger \& Freund (2004) erhoben. Mit jeweils zwei Items beantworteten die Teilnehmenden, wie sehr ihre körperliche Aktivität mit ihrem Ziel, sich gesund zu ernähren, in Konflikt steht und umgekehrt (Zielkonflikt). Mit jeweils zwei weiteren Items beantworteten sie, wie sehr ihre körperliche Aktivität ihr Ziel, sich gesund zu ernähren fördert und umgekehrt (Zielkohärenz). Zwei Beispiel-Items sind die folgenden: „Meine körperliche Aktivität fördert meine gesunde 
Ernährung.“, „,Meine gesunde Ernährung hemmt meine körperliche Aktivität.“. Die Teilnehmenden wurden gebeten, ihre Zustimmung zu diesen Items auf jeweils einer Skala von 1 bis 4 (stimmt nicht - stimmt genau) zu geben.

\subsection{Untersuchungsdesign und Intervention}

Es wurde eine Längsschnitt-Internetstudie mit zwei Messzeitpunkten im Abstand von vier Wochen durchgeführt. Die Teilnehmenden füllten einen Internetfragebogen zu den Themen ,körperliche Aktivität“" und „Obst- und Gemüseverzehr“ zum ersten Messzeitpunkt aus. Einen Monat nach der ersten Befragung erhielten die Teilnehmenden eine Einladung zum zweiten Teil der Studie (wie oben beschrieben wurde nur die Substichprobe, die den ersten Fragebogen eine Woche nach Freischaltung und den zweiten Fragebogen vier Wochen später beantwortet hat für die Analysen für Hypothese 4 herangezogen; alle anderen Analysen beziehen sich auf die Gesamtstichprobe zum Messzeitpunkt 1).

Zum ersten Messzeitpunkt wurde jeder Teilnehmende mithilfe eines Algorithmus der Software dynQuest (Rademacher \& Lippke, 2007) zufällig einer von drei Gruppen zugeordnet: Die ersten zwei Gruppen waren Interventionen (Risiko- und Planungsintervention), die dritte Gruppe war die Kontrollgruppe. Teilnehmende in der Planungsintervention sollten sich ein Verhalten aussuchen und dieses dann genauer planen (Bewegung oder Obst- und Gemüseverzehr). Teilnehmende wurden in der Intervention aufgefordert, genau aufzuschreiben, wann, wie und wo sie ihr Zielverhalten ausüben möchten, um damit Implementationsintentionen $\mathrm{zu}$ bilden, welche sich förderlich auf die Initiierung und Aufrechterhaltung einer Handlung auswirken (Gollwitzer, 1996). In der Risikointervention erhielten Teilnehmende ein Quiz mit drei Fragen zu Obst- und Gemüseverzehr und körperlicher Aktivität. Dabei wurde explizit auf die Risiken von obst- und gemüsearmer Ernährung und körperlicher Inaktivität hingewiesen, auch wenn die Fragen des Quizzes positiv formuliert waren. Die Teilnehmenden sollten für vier Fragen jeweils die Richtige aus mehreren Möglichkeiten auswählen (zum Beispiel ,Welche der unten genannten Krankheiten können eine Folge von Bewegungsmangel und falscher Ernährung sein?“). Nach jeder Frage erhielten die Teilnehmenden Rückmeldung, ob sie richtig lagen. Die Rückmeldungen hoben erneut Risiken von falscher Ernährung und Bewegungsmangel hervor. Die Teilnehmenden der Kontrollgruppe wurden lediglich nach ihrer Zielsetzung gefragt: Sie sollten angeben, welche Obst- und Gemüsesorten sie regelmäßig essen möchten und welche körperliche Aktivität sie ausüben möchten. Darüber hinaus wurden Teilnehmende in der Kontrollgruppe weder explizit gebeten, ihr Verhalten zu planen noch explizit auf Risiken ungesunder Ernährung und Inaktivität hingewiesen. Die Frage der Zielsetzung erhielten auch Teilnehmende der beiden Interventionsgruppen.
Die Risikointervention ist theoretisch nach dem HAPA stadienpassend für Non-Indender. Die Planungsintervention ist - ebenfalls nach dem HAPA - stadienpassend für Intender. Da aus untersuchungstechnischen Gründen keine für $A c$ tor stadienpassende Intervention angeboten wurde, wurden als Actor klassifizierte Teilnehmende in eine der oben genannten Interventionen randomisiert. Allerdings wurden $A c$ tor aus diesem Grund aus den längsschnittlichen Analysen ausgeschlossen, in denen die Effekte von stadienpassenden Interventionen auf Zielkonflikte getestet wurden. Dies geschah vor allem, um die Komplexität des Studiendesigns zu reduzieren.

\subsection{Statistische Auswertung}

Die Datenauswertung erfolgte mit dem Statistikprogramm SPSS (Version 13.0 für Windows). Es wurden Korrelationen und Varianzanalysen mit post hoc Tests gerechnet. Die Analysen für die Hypothesen 1 bis 3 beziehen sich dabei auf den Messzeitpunkt 1. Analysen für die Hypothese 4 beziehen sich auf beide Messzeitpunkte. Alle deskriptiven Statistiken der in dieser Untersuchung erhobenen Variablen sind in Tabelle 1 aufgeführt.

Personen, die vor der Zuweisung zu einer Interventionsgruppe beim ersten Messzeitpunkt die Teilnahme abgebrochen haben, wurden von allen weiteren Analysen ausgeschlossen. Dies betraf $18 \%(n=278)$ der Ausgangsstichprobe. Von den anderen Teilnehmenden beantworteten $99 \%$ mehr als $50 \%$ des Fragebogens.

\section{Ergebnisse}

\subsection{Zusammenhang zwischen den beiden Gesundheitszielen (Hypothese 1)}

Intentionen. Mithilfe von Korrelationsanalysen wurde der Zusammenhang zwischen den Intentionen, körperliche Aktivität auszuüben und fünf Portionen Obst und Gemüse zu essen, ermittelt. Die Intention, fünf Portionen Obst und Gemüse zu essen, korrelierte niedrig, jedoch statistisch bedeutsam mit der Intention, anstrengende körperliche Aktivität auszuüben $(r=0.18, p<0.01)$, ebenfalls niedrig, aber statistisch bedeutsam mit der Intention, mittelmäßig anstrengende Aktivität auszuüben $(r=0.16, p<0.01)$ und wiederum niedrig, jedoch auch statistisch bedeutsam mit der Intention, leichte körperliche Aktivität auszuüben $(r=0.13, p<0.01$; durchschnittliche Korrelation von $r=0.16, p<0.05)$.

Verhalten. Auch das Verhalten, körperlich aktiv zu sein und regelmäßig Obst und Gemüse zu essen, korrelierte signifikant, wenn auch niedrig $(r=0.16, p<0.001)$. Teilnehmende, die der Frage, ob sie regelmäßig Obst- und Gemüse essen, stärker zustimmten, gaben auch eine häufigere Dauer von anstrengender oder mittelmäßig anstrengender körperli- 


\begin{tabular}{|c|c|c|c|c|c|}
\hline & $M Z P$ & $M$ & $S D$ & Skala & Items \\
\hline \multicolumn{6}{|l|}{ Intentionen } \\
\hline Körperliche Aktivität & $\mathrm{T} 1$ & 2.51 & 1.10 & $1-4$ & 1 \\
\hline leicht & $\mathrm{T} 1$ & 2.51 & 1.10 & $1-4$ & 1 \\
\hline mittelmäßig anstrengend & $\mathrm{T} 1$ & 2.86 & 0.92 & $1-4$ & 1 \\
\hline anstrengend & $\mathrm{T} 1$ & 2.63 & 1.03 & $1-4$ & 1 \\
\hline Obst- und Gemüseverzehr a & $\mathrm{T} 1$ & 2.62 & 0.81 & $1-4$ & 1 \\
\hline \multicolumn{6}{|l|}{ Verhalten } \\
\hline Körperliche Aktivität ${ }^{\mathrm{b}}$ & $\mathrm{T} 1$ & 177.08 & 187.11 & $0-1050$ & 2 \\
\hline Ernährung: Berichtete Portionen & $\mathrm{T} 1$ & 1.34 & 0.91 & $0-15$ & 1 \\
\hline Ernährung: Item ${ }^{c}$ & $\mathrm{~T} 1$ & 2.55 & 1.10 & $1-4$ & 1 \\
\hline \multicolumn{6}{|l|}{ Zielkohärenz } \\
\hline Bewegung fördert Ernährung & $\mathrm{T} 1$ & 2.86 & 0.96 & $1-4$ & 1 \\
\hline T22.850.901-41 & & & & & \\
\hline $\begin{array}{l}\text { Ernährung fördert Bewegung } \\
\text { T22.910.951-41 }\end{array}$ & T1 & 2.82 & 0.95 & $1-4$ & 1 \\
\hline \multicolumn{6}{|l|}{ Zielkonflikt } \\
\hline $\begin{array}{l}\text { Bewegung hemmt Ernährung } \\
\text { T21.370.641-41 }\end{array}$ & T1 & 1.44 & 0.71 & $1-4$ & 1 \\
\hline $\begin{array}{l}\text { Ernährung hemmt Bewegung } \\
\text { T21.290.541-41 }\end{array}$ & $\mathrm{T} 1$ & 1.31 & 0.58 & $1-4$ & 1 \\
\hline \multicolumn{6}{|l|}{ Stadien } \\
\hline & & $N-I^{*}$ & $l^{*}$ & $A^{*}$ & \\
\hline Körperliche Aktivität & $\mathrm{T} 1$ & $24 \%$ & $27 \%$ & $49 \%$ & \\
\hline Obst- und Gemüseverzehr & T1 & $41 \%$ & $25 \%$ & $34 \%$ & \\
\hline
\end{tabular}

Tabelle 1: Mittelwerte $(M)$ und Standardabweichungen $(S D)$ der verwendeten Skalen und Items.

$M Z P=$ Messzeitpunkt, Skala = Antwortoptionen, Items = Anzahl der Items; a Intention, täglich fünf Portionen Obst und Gemüse zu essen; b Summenscore (anstrengende und mittelmäßige körperliche Aktivität auszuüben, in Minuten pro Woche); c Itemwortlaut: „Ich habe in der letzten Woche fünf Portionen Obst und Gemüse täglich gegessen“; * $N$ $I=$ Non-Intenders, $I=$ Intenders, $A=$ Actors. cher Aktivität pro Woche an. Die beiden Verhaltensweisen Bewegung und Ernährung hängen demnach positiv miteinander zusammen

\subsection{Berichten Teilnehmende mehr Zielkohärenz als Zielkonflikt? (Hypothese 2)}

Hypothese 2 postulierte, dass die Teilnehmenden signifikant mehr Kohärenz zwischen den beiden Zielen als Konflikt berichten. Dies wurde mit Hilfe von Mittelwertsvergleichen zu Messzeitpunkt 1 getestet. Auf die Frage, ob Bewegung die gesunde Ernährung fördert oder hemmt, berichteten die Teilnehmenden signifikant mehr Kohärenz $(M=2.86, S D=$ $0.96)$ als Konflikt $(M=1.44, S D=0.71, t(1237)=-31.71$, $p<0.001, r=-0.17)$. Dies traf auch auf die Frage $\mathrm{zu}, \mathrm{ob}$ die Ernährung die Bewegung fördert oder hemmt. Wieder berichteten die Teilnehmenden signifikant mehr Kohärenz $(M=2.82, S D=0.95)$ als Konflikt $(M=1.31, S D=0.58$; $t(1231)=-45.74, p<0.001, r=-0.09)$.

\subsection{Erlebte Zielkohärenz und Zielkonflikte in den} verschiedenen Stadien (Hypothese 3)

Zielkohärenz. Die Teilnehmenden wurden gebeten einzuschätzen, wie sehr 1. ihre Bewegung ihre Ernährung fördert und 2. ihre Ernährung ihre Bewegung fördert. Die Analysen zur erlebten Zielkohärenz in den verschiedenen Stadien (Hypothese 3) bezogen sich dementsprechend auf beide Items separat. Um die Teilnehmenden einem Stadium zuzuordnen, wurden für die erste Analyse (,Bewegung fördert Ernährung“) die Angaben zur körperlichen Aktivität und für die zweite Analyse (,Ernährung fördert Bewegung") die Angaben zum Obst- und Gemüseverzehr verwendet.

Ob Personen im non-intentionalen Stadium (NonIntender) signifikant weniger Kohärenz als Personen im intentionalen oder aktiven Stadium (Intender und Actor) erleben, wurde mittels einer ANOVA überprüft. Dabei zeigte sich ein signifikanter Haupteffekt für Stadium $(F(2,1235)=$ 22.01, $p<0.001, \eta^{2}=0.03$, für Item „Bewegung fördert Ernährung" und $F(2,1231)=25.31, p<0.001, \eta^{2}=0.04$, für Item „Ernährung fördert Bewegung“). Hypothesenkonform berichteten Non-Intender am wenigsten Zielkohärenz. Posthoc Analysen zeigten, dass Non-Intender sich in ihrer Wahrnehmung von Zielkohärenz signifikant von Intenders und $A c$ tors unterschieden; letztere beiden Gruppen unterschieden sich nicht signifikant (Mittelwerte und Konfidenzintervalle siehe Abbildung 1; post hoc-Tests siehe Tabelle 2).

Zielkonflikt. Analog zu den Einschätzungen zur Zielkohärenz wurden die Teilnehmenden gebeten, ihre Zielkonflikte einzuschätzen. Auch hier wurden zwei getrennte Analysen gerechnet: 1. Bewegung hemmt Ernährung (mit dem Stadium für körperliche Aktivität) und 2. Ernährung hemmt Bewegung (mit dem Stadium für Obst- und Gemüseverzehr). Es wurde getestet, ob Non-Intender auch mehr Zielkonflikt berichten als Personen im intentionalen oder akti- 


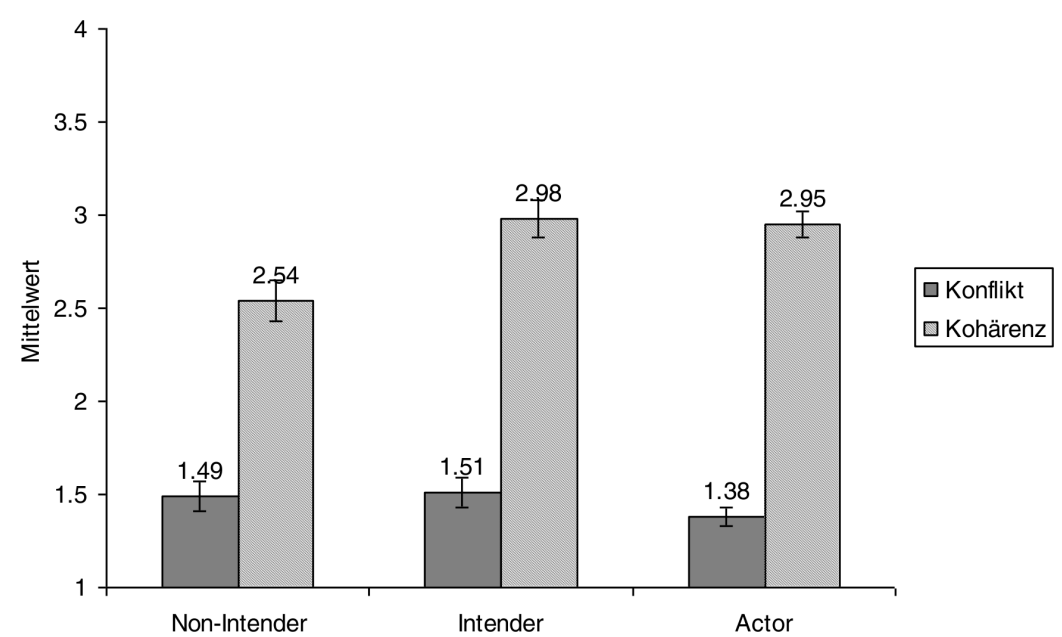

Abbildung 1: Mittelwerte und Konfidenzintervalle der wahrgenommenen Zielkonflikte und Zielkohärenz für den Einfluss von Bewegung auf Ernährung für die Teilnehmenden in den drei HAPA Stadien (Antwortskala 1 - stimmt nicht bis 4 - stimmt genau). ven Stadium (Intender und Actor). Wieder zeigte sich ein signifikanter Haupteffekt für Stadium $(F(2,1231)=4.52$, $p<0.01, \eta^{2}=0.01$, für Item ,Bewegung hemmt Ernährung" and $F(2,1232)=9.88, p<0.001, \eta^{2}=0.02$, für Item „Ernährung hemmt Bewegung“). Post-hoc-Analysen zeigten, dass Non-Intender und Intender zwar am meisten Konflikte berichteten, sich jedoch nicht signifikant voneinander unterschieden (unabhängig von der Richtung des Items). Für das Item „Ernährung hemmt Bewegung“ unterschieden sich Non-Intenders jedoch signifikant von Actors, für das Item „Bewegung hemmt Ernährung“ verfehlte das Ergebnis dieses Gruppenvergleichs das Signifanzniveau von 0.05 knapp $(p=0.07)$. Der Unterschied zwischen Intenders und Actors war für beide Richtungen jeweils signifikant (Mittelwerte und Konfidenzintervalle siehe Abbildung 2; post hoc-Tests siehe Tabelle 2).

\subsection{Stadienpassende Interventionen und Zielkonflikte (Hypothese 4)}

Zur Beantwortung der Hypothese 4 wurde auf die Teilnehmenden zurückgegriffen, die an beiden Messzeitpunkten teilgenommen hatten $(n=300)$. Von diesen $n=300$ Teilnehmenden wurden jedoch all diejenigen ausgeschlossen, die sich bereits im aktionalen Stadium für eines der beiden Verhaltensweisen befanden, da es in der Hypothese um den Effekt von stadienspezifischen Interventionen ging und in der Studie aus untersuchungstechnischen Gründen keine stadienpassende Intervention für Actors angeboten wurde. Analysiert wurden jeweils die wahrgenommenen Konflikte zwischen körperlicher Aktivität und Ernährung $(n=115)$ und zwischen Ernährung und körperlicher Aktivität $(n=119)$ getrennt (abhängige Variable). Als Gruppierungsvariable wurde eine neue Variable erstellt, die für jede Person angab, ob sie eine stadienpassende (2), eine stadienunpassende (1) oder keine Intervention $(0)$ erhalten hatte. Im Folgenden werden nur die Ergebnisse für die Hypothese, dass stadienpassende
Interventionen (für Intender die Planungsintervention beziehungsweise für Non-Intender die Risikointervention) effektiver sind als stadienunpassende berichtet. Mithilfe geplanter Kontraste wurde getestet, ob stadienpassende Interventionen nach einem Zeitpunkt von vier Wochen Zielkonflikte signifikant stärker reduzierten als stadienunpassende Interventionen.

Zielkonflikt zwischen Bewegung und gesunder Ernährung. Die Ergebnisse für das Item „Bewegung hemmt Ernährung" zeigten keinen signifikanten Haupteffekt von Zeit (von T1 zu T2, $F(1,121)=1.14, n s)$ und Interventionsgruppe $(F(2,121)=0.37, n s)$. Die Mittelwerte waren bei T1 $M=1.44(S D=0.71)$ und bei T2 $M=1.37(S D=0.64)$. Allerdings erwies sich die Interaktion zwischen Zeit und Interventionsgruppe als signifikant $(F(2,121)=3.74, p<0.05$, $\left.\eta^{2}=0.06\right)$. Das heißt die Veränderung von wahrgenommenen Konflikten unterschied sich über die Zeit zwischen den Gruppen. Ein geplanter Kontrast zeigte, dass stadienpassende Interventionen Zielkonflikte signifikant stärker reduzierten als stadienunpassenden Interventionen $(t(121)=2.45$, $p=0.016, \eta^{2}=0.05$, vergleiche Abbildung 3$)^{2}$.

Zielkonflikt zwischen gesunder Ernährung und Bewegung. Die signifikante Interaktion zwischen Interventionsgruppe und Zeit zugunsten der stadienpassenden Interventionen konnte für den wahrgenommenen Zielkonflikt zwischen gesunder Ernährung und Bewegung nicht repliziert werden. Die Ergebnisse zeigten keine signifikanten Haupteffekte für Interventionsgruppe $(F(2,165)=0.07, p=0.93)$ und keine signifikante Interaktion zwischen Interventionsgruppe und Zeit $(F(2,165)=0.31, p=0.73)$. Stattdessen zeigte sich ein signifikanter Haupteffekt für Zeit $(F(1,165)=6.48$,

\footnotetext{
${ }^{2}$ Personen in der Kontrollgruppe ohne Intervention wurde für diese Analysen nicht berücksichtigt. In einer weiteren Analyse wurden stadienpassende Interventionen mit stadienunpassenden und keinen Interventionen kontrastiert. Die Ergebnisse zeigten, dass stadienpassende Interventionen Zielkonflikte auch signifikant stärker als stadienunpassende oder keine Interventionen reduzierten $\left(t(121)=2.73, p<0.01, \eta^{2}=0.06\right)$.
} 

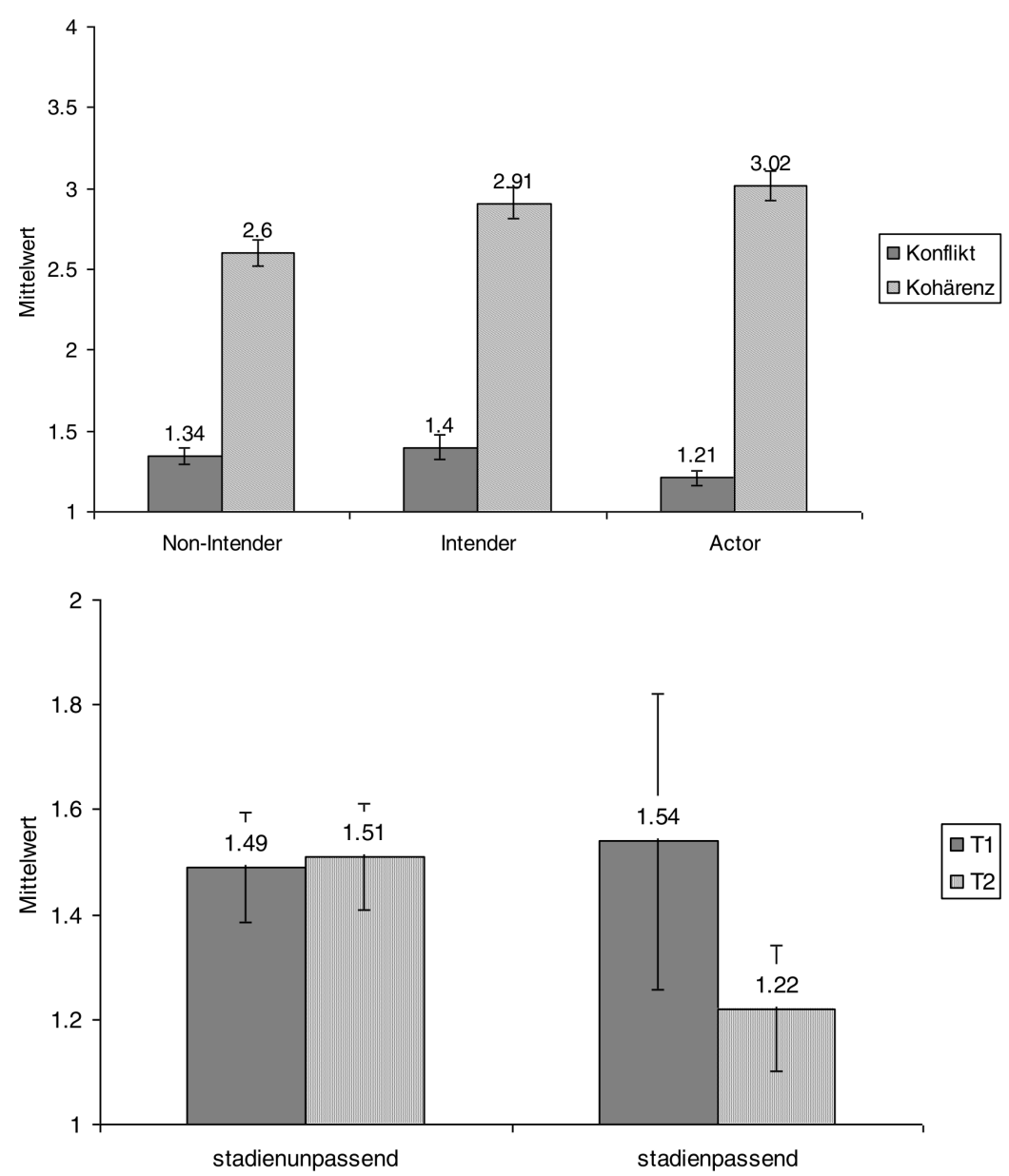

Abbildung 2: Mittelwerte und Konfidenzintervalle der wahrgenommenen Zielkonflikte und Zielkohärenz für den Einfluss von Ernährung auf Bewegung für die Teilnehmenden in den drei HAPA Stadien (Antwortskala 1 - stimmt nicht bis 4 - stimmt genau).

\begin{abstract}
Abbildung 3: Mittelwerte und Konfidenzintervalle der wahrgenommenen Zielkonflikte zum Messzeitpunkt T1 und T2 für das Item Zielkonflikt zwischen Bewegung und Ernährung jeweils für die Gruppe mit stadienpassender Intervention und stadienunpassender Intervention (Antwortskala 1 - stimmt nicht bis 4 - stimmt genau).
\end{abstract}

$\left.p=0.012, \eta^{2}=0.04\right)$. Die Teilnehmenden gaben weniger Zielkonflikte zu T2 an $(M=1.29, S D=0.54)$ als zu T1 $(M=1.31, S D=0.58)$.

\subsection{Zusammenfassung}

Es konnte gezeigt werden, dass (1) körperliche Aktivität und Obst- und Gemüseverzehr positiv miteinander korrelieren und sich eher gegenseitig fördern als behindern. Ferner schienen sich (2) wahrgenommene Konflikte über die Stadien hinweg zu unterscheiden: Personen im intentionalen Stadium (Intender) nahmen am meisten Konflikte wahr. Auch die wahrgenommene Kohärenz unterschied sich zwischen den Stadien: Teilnehmende im non-intentionalen Stadium (Non-Intender) berichteten am wenigsten Kohärenz; handelnde Personen (Actor) hingegen am meisten. Schließlich zeigte sich, dass (3) die wahrgenommenen Zielkonflikte von Teilnehmenden, die eine stadienpassende Interventionen erhalten hatten, über die Zeit stärker abnahmen als die wahrgenommenen Zielkonflikte von Teilnehmenden, die eine stadienunpassende Interventionen erhalten hatten. Dies konnte jedoch nur für den wahrgenommenen Einfluss von körperlicher Aktivität auf eine gesunde Ernährung gezeigt werden und nicht umgekehrt.

\section{Diskussion}

Gesundheitsförderung, allen voran die meisten Präventionsmaßnahmen gegen Übergewicht, setzt häufig an körperlicher Aktivität und gesunder Ernährung an. Im Sinne der Förderung einer gesunden Lebensweise untersuchte die vorliegende Studie das Verhältnis dieser beiden Gesundheitsverhalten. Ziel der Studie war es nicht, eine ganzheitliche theorie- und evidenzbasierte Maßnahme zu entwickeln, sondern Hinweise für die Gestaltung von Interventionen speziell in Bezug auf mögliche Zielkonflikte zu geben. Dafür griff die vorliegende Studie auf das Sozial-Kognitive Prozessmodell des Gesundheitsverhaltens (Health Action Process Approach; HAPA; Schwarzer, 2008) und auf Zieltheorien (Riediger \& Freund, 2004; Riediger et al., 2005) zurück.

Wie erwartet hängen die Gesundheitsziele, körperlich aktiv zu sein und viel Obst und Gemüse zu essen, positiv miteinander zusammen. Die Zusammenhänge sind zwar gering, allerdings war in dieser Studie besonders die Richtung von Bedeutung. Darüber hinaus konnte die Studie die Ergebnisse anderer Studien replizieren, die Zusammenhänge ähnlicher Größenordnung fanden (vergleiche Quintiliani et al., 2010; Werch et al., 2010; Keller et al., 2008; Verkooijen et al., 2008). Dass sich die beiden Verhaltensweisen eher ge- 
genseitig fördern als behindern, bestärkt die Hypothese, dass Interventionen, die an beiden Verhaltensweisen gleichzeitig ansetzen, von „synergistischen Effekten“ (Vandelanotte et al., 2005) profitieren könnten. Demnach scheint es sinnvoll, in Interventionen sowohl körperliche Aktivität als auch Ernährung simultan anzusprechen (Vandelanotte et al., 2007), mehr Forschung ist jedoch nötig, um diese Schlussfolgerung zuzulassen.

Bemerkenswert ist der Befund, dass Personen im nonintentionalen Stadium zwar, wie vorhergesagt, am wenigsten Kohärenz, nicht aber signifikant mehr Konflikte als Personen im intentionalen Stadium berichteten. Intender nahmen sogar mehr Konflikte wahr als Non-Intender, allerdings wurde der Unterschied nicht signifikant. Rückblickend erscheint es plausibel, dass gerade Personen, die sich an der Schwelle zur Umsetzung ihres Zielverhaltens befinden (Intender), besonders viele Konflikte wahrnehmen. Ist der erste Schritt erstmal getan, nehmen die Konflikte wieder ab (Actor). Das bedeutet aber auch, dass Interventionen bei Personen, die sich gerade an der Schwelle zur aktiven Verhaltensänderung befinden, mögliche Zielkonflikte thematisieren sollten. Dies könnte beispielsweise geschehen, indem Teilnehmende gebeten werden, ihre wichtigsten Ziele aufzuschreiben und konfligierende Ziele zu identifizieren. Im Rahmen der Intervention sollten dann mögliche Lösungen besprochen werden, wie die Ziele trotzdem erreicht werden können.

Eine weitere Frage war, ob Interventionen dazu beitragen können, die wahrgenommenen Konflikte zu reduzieren. Es konnte gezeigt werden, dass Teilnehmende, die eine stadienpassende Intervention zum ersten Messzeitpunkt erhalten hatten, zum zweiten Messzeitpunkt signifikant weniger wahrgenommene Zielkonflikte berichteten als Teilnehmende, die zum ersten Messzeitpunkt eine unpassende Intervention erhalten hatten. Dieser Befund konnte jedoch nur für den Einfluss von körperlicher Aktivität auf gesunde Ernährung gezeigt werden. Für gesunde Ernährung wurde die Interaktion zwischen Intervention und Zeit nicht signifikant. Ein Grund dafür könnte methodologischer Natur sein. Anders als körperliche Aktivität, die konsequent definiert war, wurde in dem eingesetzten Fragebogen an manchen Stellen von Obst- und Gemüseverzehr gesprochen, an anderen Stellen nur von gesunder Ernährung. Dies kann die Teilnehmenden irritiert und zu inkonsistenten Ergebnissen geführt haben. Mehr Forschung ist jedoch notwendig, um die genauen Mechanismen, durch welche stadienpassende Interventionen dazu beitragen Zielkonflikte zu reduzieren, zu identifizieren. Napolitano und Kollegen (Napolitano et al., 2008) argumentieren, dass die theoretischen Konstrukte sozialkognitiver Modelle ihrerseits als Mediatoren fungieren und die Effekte von Interventionen auf Verhalten beeinflussen können. In diesem Sinne könnten Zielkonflikte selbst zu Erfolg oder Misserfolg einer Intervention beitragen (vergleiche auch Lewis et al., 2002).
Ein weiterer Aspekt der Studie ist der Versuch, das Internet als Interventionsmedium zu nutzen. Ein Vorteil internetbasierter Gesundheitsförderung wäre ihre lokale Unbegrenztheit (Rademacher \& Lippke, 2007). Aus diesem Grund wurden die Teilnehmenden um ihre Ortsangabe gebeten. Die Ortsangaben verdeutlichen, dass die Teilnehmenden über das gesamte Bundesgebiet verteilt waren, obwohl für die Studie hauptsächlich in Berlin und Umgebung geworben wurde. Zukünftig könnte das Internet benutzt werden, um Menschen zu gewinnen, in deren Umgebung die Unterstützungsangebote noch nicht ausprägt sind. Natürlich haben internetbasierte Interventionsmaßnahmen auch Nachteile. Anders als in Trainingsprogrammen und Beratungsgesprächen findet kein persönlicher Austausch statt und die Teilnehmenden müssen sich stärker selbst motivieren. Dies könnte das Internet für manche Menschen unattraktiver machen. Darüber hinaus haben Studien gezeigt, dass die Internetnutzung mit zunehmendem Bildungsniveau steigt (siehe unten). Allerdings ist anzunehmen, dass sich dies mit weiterer Verbreitung des Internets ändern wird. Mehr Forschung ist jedoch notwendig, um die speziellen Vor- und Nachteile von Internetinterventionen zu beleuchten.

\subsection{Grenzen der vorliegenden Studie}

Eine Einschränkung der vorliegenden Studie besteht darin, dass sowohl die Angaben zur körperlichen Aktivität als auch zur Ernährung auf Selbstbericht beruhen. Insbesondere bei körperlicher Aktivität kann dies zu einer Überschätzung des Verhaltens führen (Gillison et al., 2006). Allerdings gibt es auch Hinweise für die Validität von selbstberichteten Angaben zur körperlichen Aktivität (Miller at al., 1994) und gesunder Ernährung (Armitage \& Conner, 1999).

Darüber hinaus wurden die wahrgenommenen Zielkonflikte und -kohärenzen nicht phasenspezifisch erhoben, sondern lauteten für Teilnehmende in allen drei Stadien gleich. Interessant wäre es, zu untersuchen, ob Zielkonflikte und -kohärenzen auch phasenspezifisch erfasst werden sollten. So könnte das Konflikt-Item für Intender folgendermaßen lauten: „Wie sehr hemmt ihre Intention, sich gesund zu ernähren, ihre Intention, körperlich aktiv zu sein“.

Eine weitere Einschränkung ist, dass die Stichprobe mit einem relativ hohen Bildungsniveau nicht repräsentativ für die deutsche Bevölkerung war. Dies könnte insofern problematisch sein, als dass Personen mit einer höheren Bildung häufig auch gesündere Verhaltensweisen aufzeigen als Personen mit geringerer Bildung (Cohen et al., 2008). Dies war auch der Fall für körperliche Aktivität: Im Durchschnitt waren die Teilnehmenden bereits zirka drei Stunden (177 Minuten) die Woche körperlich aktiv. Allerdings hatte fast die Hälfte der Teilnehmenden noch einen BMI von über 25, und gehört damit zur Zielgruppe von Gesundheitsförderungsmaßnahmen. Hinzu kommt, dass die Ergebnisse für den Obst- und Gemüseverzehr anders aussahen: Im Durchschnitt 


\begin{tabular}{|c|c|c|c|c|c|}
\hline & & & $\begin{array}{l}\text { Mittelwerts- } \\
\text { unterschiede }\end{array}$ & $S E$ & $p$ \\
\hline $\begin{array}{l}\text { Zielkohärenz } \\
\text { 1) Bewegung }\end{array}$ & $\begin{array}{l}\text { Non-intender } \\
\text { Intender } \\
\text { Actor }\end{array}$ & $\begin{array}{l}\text { Intender } \\
\text { Actors } \\
\text { Non-intender } \\
\text { Actor } \\
\text { Non-intender } \\
\text { Intender }\end{array}$ & $\begin{array}{l}-0.44^{\star *} \\
-0.41^{* *} \\
0.44^{* *} \\
0.03 \\
0.41^{\star *} \\
-0.03\end{array}$ & $\begin{array}{l}0.08 \\
0.07 \\
0.08 \\
0.06 \\
0.07 \\
0.06\end{array}$ & $\begin{array}{c}<0.001 \\
<0.001 \\
<0.001 \\
0.89 \\
<0.001 \\
0.89\end{array}$ \\
\hline $\begin{array}{l}\text { Zielkohärenz } \\
\text { 2) Ernährung }\end{array}$ & $\begin{array}{l}\text { Non-intender } \\
\text { Intender } \\
\text { Actor }\end{array}$ & $\begin{array}{l}\text { Intender } \\
\text { Actor } \\
\text { Non-intender } \\
\text { Actor } \\
\text { Non-intender } \\
\text { Intender }\end{array}$ & $\begin{array}{l}-0.31^{\star \star} \\
-0.42^{\star \star} \\
0.31^{\star \star} \\
-0.11 \\
0.42^{\star *} \\
0.11\end{array}$ & $\begin{array}{l}0.07 \\
0.06 \\
0.07 \\
0.07 \\
0.06 \\
0.07\end{array}$ & $\begin{array}{c}<0.001 \\
<0.001 \\
<0.001 \\
0.25 \\
<0.001 \\
0.25\end{array}$ \\
\hline $\begin{array}{l}\text { Zielkonflikt } \\
\text { 1) Bewegung }\end{array}$ & $\begin{array}{l}\text { Non-intender } \\
\text { Intender } \\
\text { Actor }\end{array}$ & $\begin{array}{l}\text { Intender } \\
\text { Actor } \\
\text { Non-intender } \\
\text { Actor } \\
\text { Non-intender } \\
\text { Intender }\end{array}$ & $\begin{array}{r}-0.01 \\
0.11 \\
0.01 \\
0.13^{\star} \\
-0.11 \\
-0.13^{\star}\end{array}$ & $\begin{array}{l}0.06 \\
0.05 \\
0.06 \\
0.05 \\
0.05 \\
0.05\end{array}$ & $\begin{array}{l}0.97 \\
0.07 \\
0.97 \\
0.03 \\
0.07 \\
0.03\end{array}$ \\
\hline $\begin{array}{l}\text { Zielkonflikt } \\
\text { 2) Ernährung }\end{array}$ & $\begin{array}{l}\text { Non-intender } \\
\text { Intender } \\
\text { Actor }\end{array}$ & $\begin{array}{l}\text { Intender } \\
\text { Actor } \\
\text { Non-intender } \\
\text { Actor } \\
\text { Non-intender } \\
\text { Intender }\end{array}$ & $\begin{array}{l}-0.06 \\
0.12^{\star \star} \\
0.06 \\
0.18^{\star \star} \\
-0.12^{\star \star} \\
-0.18^{\star \star}\end{array}$ & $\begin{array}{l}0.05 \\
0.04 \\
0.05 \\
0.04 \\
0.04 \\
0.04\end{array}$ & $\begin{aligned} & 0.40 \\
& 0.001 \\
& 0.40 \\
< & 0.001 \\
& 0.001 \\
< & 0.001\end{aligned}$ \\
\hline
\end{tabular}

Tabelle 2: Gruppenmittelwertsvergleiche (Post-hoc-Test: Games-Howell) für die drei HAPA Stadien (Bewegung beziehungsweise Ernährung). Abhängige Variablen: Zielkohärenz, 1) Körperliche Bewegung fördert gesunde Ernährung, 2) Gesunde Ernährung fördert körperliche Bewegung; und Zielkonflikt, 1) Körperliche Bewegung hemmt gesunde Ernährung, 2) Gesunde Ernährung hemmt körperliche Bewegung.

$S E=$ Standardfehler

gaben die Teilnehmenden an, nur ein bis zwei Portionen Obst oder Gemüse am Tag zu essen statt der empfohlenen fünf Portionen. Darüber hinaus war die Stichprobe hinsichtlich ihrer Bildung repräsentativ für die Gruppe der Internetnutzer, denn einer Umfrage von Emnid zufolge steigt die Internetnutzung mit Bildung und sozioökonomischen Status (Sietmann, 2002).

Die Studie erfasst die Intentionen und das selbstberichtete Verhalten im Bereich Ernährung und körperliche Bewegung über einen Zeitraum von vier Wochen. Wie die Ergebnisse gezeigt haben, können Teilnehmende bereits in dieser relativ kurzen Zeit ihr Verhalten ändern. Allerdings gibt die Studie keine Hinweise, ob diese Veränderung auch dauerhaft beibehalten werden kann (vergleiche auch Ayyad \& Andersen, 2000 für eine Metaanalyse zu den Langzeiteffekten von Ernährungsumstellungen bei der Behandlung von Adipositas). Langzeitstudien, die über einen Monat hinausgehen, sind daher notwendig, um über langfristige Effekte Aussagen treffen zu können.

Trotz dieser Einschränkungen hat die Studie sowohl praktische als auch theoretische Konsequenzen. Wie eingangs beschrieben, steigen die Kosten für das Gesundheitssystem, die auf ungesunde Ernährung und körperliche Inaktivität zurückzuführen sind (vergleiche auch Zahlen des Statistischen Bundesamtes, 2008). Daher ist es besonders wichtig, effektive und kostengünstige Gesundheitsförderungsmaßnahmen zu finden, die sowohl das Ernährungs- als auch das Bewegungsverhalten gleichzeitig ansprechen und fördern können. Die Bedeutung der Studie liegt in der Verbindung von Forschung zu Gesundheitsverhaltensänderung mit Erkenntnissen über Zielkonflikte. Wie frühere Forschung bereits gezeigt hat, können Zielkonflikte die Zielerreichung behindern und sogar zum Aufgeben der Ziele führen (vergleiche Riediger \& Freund, 2004). Doch gerade im Bereich körperlicher Aktivität und gesunder Ernährung kann das Aufgeben der persönlichen Ziele schwerwiegende gesundheitliche Folgen haben. Die Ergebnisse der Studie unterstützen das Vorgehen von Gesundheitsförderungsmaßnahmen, mehrere Verhaltensweisen gleichzeitig anzugehen. Sie zeigen jedoch auch, dass Zielkonflikte zwischen Gesundheitszielen existieren und den Zielfortschritt behindern können. Dies sollte im Rahmen von Gesundheitsförderung thematisiert werden, indem beispielsweise persönliche Zielkonflikte angesprochen werden. Weitere Forschung ist nötig, um die genauen Wirkmechanismen von Zielkonflikten zwischen Gesundheitszielen zu untersuchen.

\section{Literatur}

Armitage, C. \& Conner, M. (1999). The theory of planned behavior: Assessment of predictive validity and 'perceived control'. British Journal of Social Psychology, 38, 35-54

Ayyad, C. \& Andersen, T. (2000). Long-term efficacy of dietary treatment of obesity: a systematic review of studies published between 1931 and 1999. Obesity Review, 1, 113-119. 
Bolognesi, M., Nigg, C. R., Massarini, M. \& Lippke, S. (2006). Reducing obesity indicators through brief physical activity counseling (PACE) in Italian primary care settings. Annals of Behavioral Medicine, 31, 179-185.

Boudreaux, E. D., Wood, K. B., Mehan, D., Scarinci, I. C., Taylor, C. L. \& Brantley, P. J. (2003). Congruence of readiness to change, self-efficacy, and decisional balance for physical activity and dietary fat reduction. American Journal of Health Promotion, 17, 329-336.

Bridle, C., Riemsma, R. P., Pattenden, J., Sowden, A. J., Mather, L., Watt, I. S. \& Walker, A. (2005). Systematic review of the effectiveness of health behavior interventions based on the transtheoretical model. Psychology \& Health, 20, 283-301.

Cohen, S., Alper, C. M., Doyle, W. J., Adler, N., Treanor, J. J. \& Turner, R.B. (2008). Objective and subjective socioeconomic status and susceptibility to the common cold. Health Psychology, 27, 268- 274.

Deutsche Gesellschaft für Ernährung. (2004). Ernährungsbericht 2004. Bonn: Deutsche Gesellschaft für Ernährung.

Fleig, L., Lippke, S., Wiedemann, A. U., Ziegelmann, J. P., Reuter, T. \& Gravert, C. (2010). Förderung von körperlicher Aktivität im betrieblichen Kontext. Ein randomisiertes Kontrollgruppen-Design zur Untersuchung von stadienspezifischen Interventionseffekten, Zeitschrift für Gesundheitspsychologie, 18, 69-78.

Gillison, F. B., Standage, M. \& Skevington, S. M. (2006). Relationship among adolescents' weight perceptions, exercise goals, exercise motivation, quality of life and leisure-time exercise behaviour: A self-determination theory approach. Health Education Research, 21, 836-847.

Godin, G. \& Shepard, R. J. (1985). A simple method to assess exercise behavior in the community. Canadian Journal of Applied Sport Science, 10, $141-146$.

Gollwitzer, P. M. (1996). The volitional benefits of planning. In P. M. Gollwitzer \& J. A. Bargh (Eds.), The psychology of action (pp. 287-312). New York: The Guilford Press.

Heckhausen, H. \& Kuhl, J. (1984). From wishes to action: The dead ends and short cuts on the long way to action. In M. Frese \& J. Sabini (Eds.), Goal-directed behavior. Hillsdale, H. J.: Erlbaum.

Kniesel, E., Ziegler, C., Lezinsky, D. \& Strang, H. (2007). Selbststeuerung und Affektregulation. Zeitschrift für Sportpsychologie, 14, 14-28.

Lewis, B. A., Marcus, B. H., Pate, R. R. \& Dunn, A. L. (2002). Psychosocial mediators of physical activity behavior among adults and children. American Journal of Preventive Medicine, 23, 26-35.

Lippke, S., Ziegelmann, J. \& Schwarzer, R. (2004). Initiation and maintenance of physical exercise: Stage-specific effects of a planning intervention. Research in Sports Medicine, 12, 221-240.

Lippke, S., Ziegelmann, J., Schwarzer, R. \& Velicer, W. F. (2009). Validity of stage assessment in the adoption and maintenance of physical activity and fruit and vegetable consumption. Health Psychology, 28, 183-198.

Miller, D. J., Freedson, P. S. \& Kline, G. M. (1994). Comparison of activity levels using the Caltrac accelerometer and five questionnaires. Medicine and Science in Sports and Exercise, 26, 376-382.

Noar, S. M., Benac, C. \& Harris, M. (2007). Does tailoring matter? Meta-analytic review of tailored print health behavior change interventions. Psychological Bulletin, 133, 673-693.

Noar, S. M., Black, H. G. \& Pierce, L. B. (2009). Efficacy of computer technology-based HIV prevention interventions: A meta-analysis. AIDS, $23,107-115$.

Napolitano, M. A., Papandonatos, G. D., Lewis, B. A., Whiteley, J. A., Williams, D. M., King, A. C., Bock, B. C., Pinto, B. \& Marcus, B. H. (2008). Mediators of physical activity behavior change: A multivariate approach. Health Psychology, 27, 409-418.

Ness, A. R. \& Powles, J.W. (1997). Fruit and vegetables, and cardiovascular disease: A review. International Journal of Epidemiology, 26, 1 13.

Ottens, M. (2005). Internetnutzung durch Privatpersonen und Unternehmen. Verfügbar unter: http://www.eds-destatis.de/de/downloads/sif /np_06_12.pdf [20. Juli 2010].

Patrick, K., Calfas, K. J., Norman, G. J., Zabinski, M. F., Sallis, J. F., Rupp, J., Covin, J. \& Cella, J. (2006). Randomized controlled trial of a primary care and home-based intervention for physical activity and nutrition behaviors: PACE+ for adolescents. Archives of Pediatrics and Adolescent Medicine, 160, 128-136.

Pomerleau, J., Lock, K., Knai, C. \& McKnee, M. (2005). Effectiveness of interventions and programmes promoting fruit and vegetable intake. Background paper for the Joint FAO/WHO workshop on fruit and vegetables for health, 1-3 September 2004, Kobe Japan. Verfügbar unter: http://www.who.int/dietphysicalactivity/publications/f\&v_promo tion_effectiveness.pdf [20. Juli 2010]

Prochaska, J. O. \& DiClemente, C. C. (1983). Stages and processes of self-change of smoking: Toward an integrative model of change. Journal of Consulting \& Clinical Psychology, 51, 390-395.

Prochaska, J. J. \& Sallis, J. F. (2004). A randomized controlled trial of single versus multiple health behavior change: Promoting physical activity and nutrition among adolescents. Health Psychology, 23, 314-318.

Puska, P., Nishida, C. \& Porter, D. (2003). Obesity and overweight: Global strategy on diet, physical activity and health. Verfügbar unter: www.who.int/dietphysicalactivity/publications/facts/obesity/en/ [19. Mai 2005].

Rademacher, J. D. M. \& Lippke, S. (2007). Dynamic online surveys and experiments with the free open-source software dynQuest. Behavior Research Methods, 39, 415-426.

Rafferty, A. P., Anderson, J. V., McGee, B. H., \& Miller, C. E. (2002). A healthy diet indicator: Quantifying compliance with the diatary guidelines using the BRFSS. Preventive Medicine, 35, 9-15.

Riediger, M. \& Freund, A. (2004). Interference and facilitation among personal goals: Differential association with subjective well-being and persistent goal pursuit. Personality and Social Psychology Bulletin, 30, 1511 1523.

Riediger, M., Freund, A. M. \& Baltes, P. B. (2005). Managing life through personal goals: Intergoal facilitation and intensity of goal pursuit in younger and older adulthood. Journals of Gerontology: Series B: Psychological Sciences \& Social Sciences, 60, pp. 84-91.

Robert Koch-Institut (Ed.). (2006). Gesundheit in Deutschland. Gesundheitsberichterstattung des Bundes. Berlin: Robert Koch-Institut.

Schwarzer, R. (2008). Modeling health behavior change: How to predict and modify the adoption and maintenance of health behaviors. Applied Psychology, 57, 1-29.

Sietmann, R. (2002). Internet-Statistik: Die digitale Spaltung erreicht die Berliner Republik. Verfügbar unter: http://www.heise.de/newsticker /meldung/27938 [14. September 2009].

Statistisches Bundesamt (2008). Statistisches Jahrbuch 2008. Bundeszentrale für Politische Bildung. Verfügbar unter: http://www.destatis.de [16. Juni 2010].

Stetina, B. U. \& Kryspin-Exner, I. (2009). Gesundheit und neue Medien. Psychologische Aspekte der Interaktion mit Informations- und Kommunikationstechnologien. Springer: Wien.

Vandelanotte, C., De Bourdeaudhuij, I. \& Brug, J. (2007). Two-year follow-up of sequential and simulaneous interactive computer-tailored interventions for increasing physical activity and decreasing fat intake. Annual Behavioral Medicine, 2007, 213-219.

Vandelanotte, C., De Bourdeaudhuij, I., Sallis, J. F., Spittaels, H. \& Brug, J. (2005). Efficacy of sequential or simultaneous interactive computertailored interventions for increasing physical activity and decreasing fat intake. Annals of Behavioral Medicine, 29, 138-146.

Weinstein, N. D., Lyon, J.E., Sandman, P. M. \& Cuite, C. L. (1998). Experimental evidence for stages of health behavior change: The precaution adoption process model applied to home radon testing. Health Psychology, $17,445-453$

Weinstein, N. D., Rothman, A. J. \& Sutton, S. R. (1998). Stage theories of health behavior: Conceptual and methodological issues. Health Psychology, 17, 290-299.

World Health Organization. (2006). Obesity and overweight. Fact Sheet No. 311. Verfügbar unter: http://www.who.int/mediacentre/factsheets /fs311/en/print.html [24. April 2009]. 